\title{
Emotion Regulation Choice: Differences in U.S. and Indian Populations
}

\author{
Ashish Mehta ${ }^{1}$, Gerald Young ${ }^{2}$, Alyssa Wicker ${ }^{3}$, Sarah Barber ${ }^{4}$, Gaurav Suri ${ }^{5}$
}

\section{ABSTRACT}

In the past two decades, researchers have conclusively demonstrated that various emotion regulation (ER) strategies give rise to differing consequences. Such findings have prompted an examination of the internal and external factors that contribute to emotion regulation choice. Previous empirical studies modeling ER choice have been limited to Western samples. Based on knowledge of the role of culture in other choice behavior, we sought to test whether culture was a driver of ER choice. For the present studies, we compared ER choices of participants from India, to ER choices of participants from the U.S.A. Research demonstrating a correlation between religiosity and effective use of cognitive reappraisal lead us to anticipate the more religious India showing higher rates of cognitive reappraisal. Based on the incorporation of acceptance themes in Indian philosophy, as well as higher rates of fatalistic outlooks in India, we also expected to see Indian participants more frequently using an acceptance ER strategy. We further expected that difference in choice strategies would be moderated by emotional intensity of the stimuli. To test these hypotheses, we presented high and low-intensity emotion-eliciting images to both samples and recorded ER choice selections. We discovered that as hypothesized, the Indian sample was significantly more likely to use cognitive reappraisal than the U.S. sample, specifically for high intensity images. Contrary to our hypothesis, the choice rate for acceptance was indistinguishable in the Indian and U.S. samples. This research indicates that culture bears considerably on which strategies people choose to employ when regulating emotion in response to negative stimuli.

Keywords: Emotion Regulation Choice, Culture, India, U.S.A.

\footnotetext{
${ }^{1}$ Department of Psychology, San Francisco State University, San Francisco, CA, U.S.A.

2 Department of Psychology, San Francisco State University, San Francisco, CA, U.S.A.

${ }^{3}$ Department of Psychology, San Francisco State University, San Francisco, CA, U.S.A.

${ }^{4}$ Department of Psychology, San Francisco State University, San Francisco, CA, U.S.A.

${ }^{5}$ Department of Psychology, San Francisco State University, San Francisco, CA, U.S.A.

*Responding Author
}

Received: February 22, 2017; Revision Received: March 19, 2017; Accepted: March 23, 2017

(C) 2017 Mehta A, Young G, Wicker A, Barber S, Suri G; licensee IJIP. This is an Open Access Research distributed under the terms of the Creative Commons Attribution License (www.creativecommons.org/licenses/by/2.0), which permits unrestricted use, distribution, and reproduction in any Medium, provided the original work is properly cited. 


\section{Emotion Regulation Choice: Differences in U.S. and Indian Populations}

Emotions are a useful adaptation to recurring challenges of human life. They allow us to form attachments and maintain relationships (Keltner \& Haidt, 1999). They prepare us physiologically to respond to potential threats (Susskind et al., 2008). They even help us to make good decisions (Bechara, Damasio, Tranel, \& Damasio, 1997). Emotions offer us non-deterministic behavioral response tendencies. However, when an emotional response is unsuited for the situation at hand, we often seek to change or regulate our emotion before translating it to a behavioral response.

A growing body of research in the past two decades has demonstrated that the choice of emotion regulation (ER) strategies we employ give rise to myriad consequences in spheres ranging from mental health, to everyday affect, to cognitive functioning, to social success (Gross \& Muñoz, 1995; English, John, Srivastava, \& Gross, 2012; Gross, 2002; Gross \& John, 2003; Richards \& Gross, 2000). Given the variety of consequences that result from choosing one ER strategy over another, it is important to understand the various factors that influence ER choice. Prior research has demonstrated that person-specific factors such as age, gender, personality, race, and available cognitive resources influence which strategies people choose to employ (Gross \& John, 2003; John \& Gross, 2004; Scheibe, Sheppes, \& Staudinger, 2015). For example, because distraction is less cognitively costly to implement, it is more likely to be chosen than reappraisal when cognitive resources are spread thin (Sheppes et al., 2014). Additionally, stimulus-specific factors such as the intensity of a negative stimulus have been shown to influence ER strategy choice. In particular, people are more likely to choose distraction over reappraisal when encountering highintensity negative situations and vice versa in low-intensity negative situations (Sheppes, Scheibe, Suri, \& Gross, 2011).

Building upon this, the present work analyzes the impact of culture on ER choice. Culture has been shown to play a role in choice behavior in many (non-ER related) domains (Weber \& Hsee, 2000; Yates \& Oliveira, 2016). However, prior research on the role of culture in ER has been subject to limitations that call for further investigation. For example, prior research has been primarily limited to Western and Far Eastern samples (Butler, Lee, \& Gross, 2007). Furthermore, many prior cross-cultural studies on ER have used self-report questionnaires given to participants regarding the strategies they use in their daily life (Kwon, Yoon, Joormann, \& Kwon, 2013; Soto,Perez, Kim, Lee, \& Minnick, 2011; Matsumoto, Yoo, \& Nakagawa, 2008). Problematically, the self-report framework is subject to issues with participants' memories, differences in ideal affect (that may influence participants' responses), and systematic situational differences confronting people in the cultures being studied. To address this limitation, in the present research, we conducted a series of studies in which ER choice was recorded immediately. This allowed for analysis of how entrenched paradigms of thought shaped by culture influence the choice of ER strategies.

To investigate this effect, we chose to compare Indian participants to American participants. We chose to focus on India for several reasons. First, despite its size, it has been understudied. India 


\section{Emotion Regulation Choice: Differences in U.S. and Indian Populations}

contains a massive $17.9 \%$ of the entire world's population ("World Population Data Sheet", 2016). This figure does not include the vast Indian diaspora consisting of another twenty million people worldwide (Safran, Sahoo, \& Lal, 2008). Second, religiosity is extraordinarily prominent in India. Recent estimates suggest that Hinduism is followed by $79.8 \%$ of the Indian population (“C-1 Population by Religious Community", 2011) and 90\% of Indians state that religion is an "important part of their daily lives" (compared to 65\% in the U.S.A.) (Crabtree, 2010). We hypothesized that the deep imbrication of religion with day to day to life in India (as compared to the U.S.A.) would impact the ER strategy choice of cognitive reappraisal. Cognitive reappraisal involves changing the meaning of a situation to reduce its emotional impact. Religion, which is a meaning-making system, has been linked to more effective use of cognitive reappraisal (Vishkin et al., 2016). Greater religiosity has also been associated with better long-term adjustment following bereavement and this superior adjustment was mediated by meaning-making coping (e.g. cognitive reappraisal; Park, 2005). Religion has also been shown to function as a coping mechanism among sick, elderly hospital patients by affording cognitive reappraisals (e.g. "God is teaching me a lesson"; Pargament, Koenig, Tarakeshwar, \& Hahn, 2004). Given that 99.1\% of Indian people identified as one of India's top six religions (" $\mathrm{C}-1$ Population by Religious Community”, 2011), and given that religion seems to foster habitual use of reappraisal, we predicted that an Indian sample would engage in reappraisal more than an American sample (Hypothesis 1).

Our second hypothesis involved choices related to the ER strategy of acceptance. Most previous studies of ER choice have focused on the strategies cognitive reappraisal and attentional distraction and have done so with participants primarily from Western cultures (Sheppes et al., 2011; Hay, Sheppes, Gross, \& Gruber, 2015; Shafir, Schwartz, Blechert, \& Sheppes, 2015). This strategy set omits acceptance-an important ER strategy that has been espoused in Indian philosophy for millennia and more recently adopted by the Western tradition with the emergence of Acceptance and Commitment Therapy (ACT; Hayes, Strosahl, \& Wilson, 1999). ACT defines acceptance as "an abandonment of dysfunctional change agendas and an active process of feeling feelings as feelings..." (Hayes et al., 1999, p. 77). Given that Hinduism advocates for acceptance of pain and suffering on the grounds of it being an inexorable aspect of life that is neither bad nor good (Whitman, 2007), we reasoned that this ER strategy would be common amongst Indian adults. This hypothesis is further supported by other research showing that fatalistic outlooks, defined as the belief that whatever happens must happen (Bernstein, 1992, p. 5), appear more frequently in India than in several other countries, including the U.S.A. (Aycan et al., 2000). In the aforementioned study, the authors found that India scored highest on a scale of fatalism as compared to 10 other countries, with the U.S.A. in the bottom 3. We expected that the presence of a fatalistic acceptance in Indian populations would result in an increased preference for acceptance as an ER strategy. 


\section{Emotion Regulation Choice: Differences in U.S. and Indian Populations}

Due to prior studies demonstrating the paramount impact of stimulus intensity on ER choice (Sheppes et al., 2011), we expected that cultural differences in the preference of reappraisal (Hypothesis 1) and acceptance (Hypothesis 2) would vary by intensity. To test these hypotheses, we used a well-validated paradigm for ER choice (Sheppes et al., 2011) to create a cross-cultural comparison of Indian and American participants. Sheppes et al. (2011) presented participants with high and low-intensity emotion-eliciting images from the International Affective Picture System and asked them to select between ER strategy options while they viewed the image again for a longer period (Lang, Bradley, \& Cuthbert, 2008). This study revealed that with images of a high-intensity negative valence, American participants were more inclined to use distraction than reappraisal. For images of a low-intensity negative valence, participants were more inclined to use reappraisal than distraction.

Since acceptance has never been studied in an ER choice context, in Study 1 we included this strategy option alongside the options to distract or reappraise. After obtaining unexpectedly high level of acceptance for low-intensity images in this study, we questioned whether participants were conflating acceptance with a decision to not use emotion regulation. To remedy this confound in Study 2, we added a "watch" option to reflect a participant's choice to simply watch the photo with no regulation. Having created and validated a new ER choice paradigm that included acceptance, in Study 3 we directly tested for differences in usage of reappraisal and acceptance between Indian and American adult samples.

\section{STUDY 1}

In Study 1, our goal was to validate an experimental structure to test ER choice between the strategies distraction, reappraisal, and the previously unstudied option of acceptance. Following prior results (Sheppes et al., 2011; Shafir et al., 2015), we expected American participants to most frequently select reappraisal for low-intensity images and infrequently for high-intensity images. We expected distraction to be chosen most frequently for high-intensity images, while being used infrequently in low-intensity images. Finally, we expected Americans to choose acceptance equally infrequently for both intensities since negative emotion is generally considered something to avoid rather than accept by American culture (Koopmann-Holm \& Tsai, 2014).

\section{Participants}

For this task, we used Amazon's Mechanical Turk survey platform (henceforth, MTurk) to recruit paid participants from across the U.SA. Thirty MTurk workers $\left(M_{\text {Age }}=40.8, S D_{\text {Age }}=\right.$ 11.06) were screened by IP address and self-report to select American-born participants, currently located in the U.S.A., that were raised by American-born parents.

\section{Procedure}

Participants were given a brief introduction to ER and then trained on how to use three common ER strategies; distraction, reappraisal, and acceptance. In this training, participants first 
previewed a negative valence image from the IAPS for one second (Lang et al., 2008). Next, they were taught how to use one of the three ER strategies. Participants practiced each strategy twice-once with a low-intensity negative image, and once with a high-intensity negative image. The order that the strategies were taught and the order that the training images were presented were both randomized.

After the training phase, participants completed two practice trials of an ER choice test adapted from Sheppes et al. (2011). Each trial consisted of previewing an image for 1 second, selecting either distraction, acceptance, or reappraisal, and then implementing their chosen strategy while they viewed the image again for 6 seconds. This was followed by a manipulation check asking participants to reaffirm the strategy they had just implemented. Image order and choice option order were counterbalanced throughout the experiment.

The 30 images used for the choice phase of the experiment were the same images used in prior studies and were chosen to create two categories of stimulus intensity (high and low) based on their normative ratings for arousal $(1=$ low; $9=$ high $)$ and valence $(1=$ very unpleasant; $9=$ highly pleasant) (Sheppes et al., 2011). The 15 low-intensity negative images were rated as mean arousal $=5.01$ and mean valence $=3.41$, while the 15 high-intensity images were rated as mean arousal $=6.12$ and mean valence $=1.99, F s(1,28)>19.01, p s<.001$. Previous studies have established that such arousal and valence differences are sufficient to create different levels of emotion-response activation, as indicated by physiological arousal (Bradley, Codispoti, Cuthbert, \& Lang, 2001) and electro cortical markers of negativity (Weinberg \& Hajcak, 2010).

The choice phase of the experiment was identical to the practice trials participants had previously completed. Participants again previewed an emotion-eliciting image for 1 second. Next, they indicated the ER strategy that would best help them to down regulate their negative emotion and implemented their chosen strategy in the subsequent 6 second viewing. Finally, participants reaffirmed which ER strategy they had actually used. Participants who failed this manipulation check on more than two trials were removed from analyses.

\section{RESULTS}

Two participants were removed from analyses for having more than two manipulation check failures. This left a final sample of 28 participants $\left(M_{\text {Age }}=41.61, S D_{\text {Age }}=10.97\right)$.

Table 1.1, ER Choice Proportions: Study 1 U.S.A.

\begin{tabular}{|l|l|l|l|l|}
\hline \multicolumn{3}{|l|}{ High Intensity } & Low Intensity \\
\hline ER Choice & $M$ & $95 \%$ CI & $M$ & $95 \%$ CI \\
\hline Acceptance & $29.52 \%$ & $19.64 \%-39.41 \%$ & $60.48 \%$ & $51.69 \%-69.26 \%$ \\
\hline Distraction & $48.33 \%$ & $38.39 \%-58.27 \%$ & $14.76 \%$ & $7.01 \%-22.51 \%$ \\
\hline Reappraisal & $22.14 \%$ & $15.47 \%-28.82 \%$ & $24.76 \%$ & $17.84 \%-31.69 \%$ \\
\hline
\end{tabular}

(C) The International Journal of Indian Psychology, ISSN 2348-5396 (e)| ISSN: 2349-3429 (p) | 206 


\section{Emotion Regulation Choice: Differences in U.S. and Indian Populations}

As expected, participants' ER choices were a function of intensity, and distraction was more frequent than both acceptance and reappraisal while viewing low-intensity images, both $t$ 's > 2.06 , both $p$ 's $<.05$, both $d$ 's $>$.38. Contrary to our hypothesis, acceptance was far more frequent than both reappraisal and distraction while viewing low-intensity images, both $t$ 's > 5.31, both $p$ 's $<.001$, both $d$ 's $>1.00$.

A 2(Intensity: High, Low) X 3(ER Strategy: Distraction, Reappraisal, Acceptance) ANOVA was conducted with both factors being within-subject variables to further examine the ER choices of participants. There was a significant Intensity X ER Strategy interaction, $F(2,54)=53.26, p<$ $.001, \eta_{p}^{2}=.66$. As illustrated in Table 1.1, participants reported using distraction more frequently while viewing high-intensity images than while viewing low-intensity images, $t(27)=8.17, p<$ $.001, d=1.54$. Participants indicated engaging in acceptance less during the presentation of highintensity images compared to low-intensity images, $t(27)=-8.34, p<.001, d=1.58$. Reappraisal usage not differ between the high and low-intensity images, $t(27)=-.89, p=.380, d=.17$.

\section{DISCUSSION}

The results reflected that participants select different ER strategies based on the image intensity. However, we were surprised by the exceedingly high rate of acceptance in the low-intensity condition since there was no conceptual reason to account for this. This lead us to hypothesize that some low-intensity images may not require emotion regulation, in which case participants might choose acceptance as a substitute for no regulation. To investigate this hypothesis, we included a "watch" option in Study 2 that would allow participants the option of not engaging in ER while viewing the images. If acceptance was being conflated with the decision to not use ER, watch would become the most frequent ER choice for low-intensity images and acceptance frequency would be reduced for low-intensity images. We tested this hypothesis in Study 2.

\section{STUDY 2}

In Study 2, we sought to finalize our experimental framework with a U.S. sample (Study 2a) and then validate that framework with an Indian pilot sample (Study 2b).

\section{Study $2 a$}

In Study 2a, we specifically sought to test whether American participants were conflating an acceptance ER strategy with a lack of need to regulate emotion. We considered that despite the average negative valence, some images may not incite every viewer to require emotion regulation. In such cases, we suspected participants would choose whichever strategy was closest to not using regulation. In Study 1, that option was acceptance. To overcome this confound in Study 2, we included a "watch" option. 


\section{Participants}

Forty-six U.S. based MTurk workers $\left(M_{\text {Age }}=40.93, S D_{\text {Age }}=13.98\right)$ were recruited to complete this survey.

\section{Procedure}

Besides the inclusion of a watch option, the survey was identical to Study 1. The newly incorporated watch option was defined to participants as an option to be used if they anticipated being able to watch the photo for the entire 6 seconds without feeling motivated to regulate their emotion. As in Study 1, choice order and picture order were counterbalanced.

Figure $2.1^{1}$

\section{Procedure: Studies 2-3a}

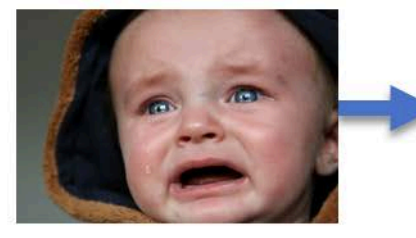

1 second

Preview

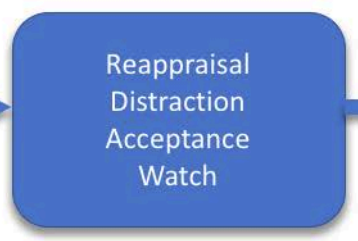

Indefinite

Select Strategy

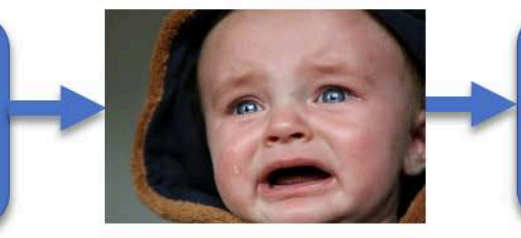

6 seconds

View while regulating
Manipulation Check

Indefinite

Reaffirm Strategy

\section{RESULTS}

The total number of failed manipulation checks was calculated identically to as in Study 1 and participants with more than two failed manipulation checks were removed from analyses. This standard led us to remove 8 participants. After these removals, a final sample of 38 was left to examine ER choices $\left(M_{\text {Age }}=40.47, S D_{\text {Age }}=13.91\right)$.

Table 2.1, ER Choice Proportions: Study 2a U.S.A.

\begin{tabular}{|l|l|l|l|l|}
\hline \multicolumn{3}{|l|}{ High Intensity } & Low Intensity \\
\hline ER Choice & $\boldsymbol{M}$ & $\mathbf{9 5 \%} \boldsymbol{C I}$ & $\boldsymbol{M}$ & $\mathbf{9 5 \%} \mathbf{C I}$ \\
\hline Acceptance & $23.68 \%$ & $17.31 \%-30.06 \%$ & $25.61 \%$ & $19.04 \%-32.19 \%$ \\
\hline Distraction & $44.91 \%$ & $36.93 \%-52.90 \%$ & $4.91 \%$ & $2.60 \%-7.23 \%$ \\
\hline Reappraisal & $23.33 \%$ & $17.64 \%-29.02 \%$ & $13.68 \%$ & $8.72 \%-18.65 \%$ \\
\hline Watch & $8.07 \%$ & $4.43 \%-11.71 \%$ & $55.79 \%$ & $45.70 \%-65.88 \%$ \\
\hline
\end{tabular}

Again, distraction was the most frequent choice for high-intensity images, all $t$ 's $>3.19$, all $p$ 's < .003 , all d's > .51.

As shown in Table 2.1, adding watch as an ER choice greatly reduced the percentage of instances participants chose to use acceptance while viewing low-intensity images. Acceptance in low-intensity conditions dropped from 60.48\% (Study 1) to 25.61\% (Study 2a). The newly created watch option was selected in $55.79 \%$ (Study 2a) of trials making it the most frequent choice for low-intensity images, all $t$ 's $>3.83$, all $p$ 's $<.001$, all $d$ 's $>.62$. This supports our

(c) The International Journal of Indian Psychology, ISSN 2348-5396 (e)| ISSN: 2349-3429 (p) | 208 


\section{Emotion Regulation Choice: Differences in U.S. and Indian Populations}

hypothesis that participants were indicating acceptance engagement when they were in fact viewing the images without using an ER strategy.

A 2(Intensity: High, Low) X 4(ER Strategy: Distraction, Reappraisal, Acceptance, Watch) ANOVA was again conducted with both factors being within-subject variables to further examine the ER choices of participants. As hypothesized, there was a significant Intensity X ER Strategy interaction, $F(3,111)=62.04, p<.001, \eta_{p}^{2}=.63$, indicating that ER choices differ between the high and low-intensity conditions. To examine the interaction, paired t-tests were used to compare ER choice selections within the high and low-intensity image types. As shown in Table 2.1, participants reported watching (i.e., no ER engagement) high-intensity images less frequently than low-intensity images, $t(37)=-11.06, p<.001, d=1.79$. Participants engagement in acceptance did not differ based on image intensity, $t(37)=-.42, p=.675, d=.07$. Participants again reported using distraction more frequently while viewing high-intensity images than lowintensity images, $t(37)=10.21, p<.001, d=1.66$. Participants reported using reappraisal more frequently while viewing high-intensity images than low-intensity images, $t(37)=3.18, p=.003$, $d=.52$.

\section{DISCUSSION}

The results of Study 2a were consistent with our hypothesis that participants were reporting engagement in acceptance when in fact they had chosen not to use ER. Participants' usage of acceptance was reduced substantially in Study 2a for low-intensity images (25.61\% from 62.72\% in Study 1) and participants reported watching with no ER 55.79\% of the time. Consistent with prior results, participants in Study 2a reported using distraction most frequently while viewing high-intensity images.

The results of Study 2a established that we had a viable experimental structure and gave us a preliminary glimpse of ER choice results with U.S. participants in a four-choice context. Our next step was to validate this structure with an Indian sample (Study 2b) and then directly compare the results between the two cultures (Study 3).

\section{Study $2 b$}

After having determined a successful structure for the ER choice survey with American participants, we subsequently sought to validate this structure on a pilot Indian sample.

\section{Participants}

Participants were screened by IP address and self-report to ensure they were currently located in India and were also born there. Twenty India based MTurk workers $\left(M_{\text {Age }}=34.05, S D_{\text {Age }}=\right.$ 10.75) were recruited to pilot the study. 


\section{Procedure}

The study was identical to that distributed in Study 2a.

\section{RESULTS}

Prior to examining the data, participants who selected more than one ER strategy following the first 1s image presentation were marked as having an "erroneous double ER choice". Those who had more than two double ER choice selections were removed because it was not possible to determine which strategy participants meant to select. This resulted in 3 participants being removed. Following the procedures of Study 1 and 2, three participants who had more than 2 failed manipulation checks were also removed. This left a final sample of 14 participants $\left(M_{\text {Age }}=\right.$ 37.07, $S D_{\text {Age }}=11.61$ ) in this pilot study.

Table 2.2, ER Choice Proportions: Study 2 b India

\begin{tabular}{|l|l|l|l|l|}
\hline \multicolumn{3}{|l|}{ High Intensity } & Low Intensity \\
\hline ER Choice & $\boldsymbol{M}$ & $\mathbf{9 5 \%}$ CI & $\boldsymbol{M}$ & $\mathbf{9 5 \%} \boldsymbol{C I}$ \\
\hline Acceptance & $24.77 \%$ & $15.28 \%-34.24 \%$ & $30.95 \%$ & $17.23 \%-44.67 \%$ \\
\hline Distraction & $32.86 \%$ & $16.77 \%-48.94 \%$ & $13.33 \%$ & $4.27 \%-22.39 \%$ \\
\hline Reappraisal & $36.19 \%$ & $23.77 \%-48.61 \%$ & $17.14 \%$ & $6.18 \%-28.10 \%$ \\
\hline Watch & $6.19 \%$ & $-0.30 \%-12.68 \%$ & $38.57 \%$ & $22.51 \%-54.63 \%$ \\
\hline
\end{tabular}

A two-way repeated measure ANOVA was conducted as in Study 1 and 2a. There was a significant Intensity X ER Strategy interaction, $F(3,39)=8.75, p<.001, \eta_{p}^{2}=.40$. Paired t-tests indicated that the Indian participants reported "watching” high-intensity images to a lesser extent than low-intensity images, $t(13)=-4.89, p<.001, d=1.31$. Participants used distraction more for high-intensity images than for low-intensity images, $t(13)=2.32, p=.038, d=.62$. Participants also used reappraisal more for high-intensity images than low-intensity images, $t(13)$ $=2.54, p=.025, d=.68$. Participants usage of acceptance did not vary by image intensity, $t(13)$ $=-1.00, p=.336, d=.27$. Within high-intensity conditions and low-intensity conditions, distraction usage and reappraisal usage did not significantly differ, both $t$ 's $<.56$, both $p$ 's $>.59$, both $d$ 's $<.15$.

\section{DISCUSSION}

The pilot indicated that the structure of the test was effective with an Indian sample. Further, there did appear to be culturally driven differences in ER choice as compared to the American sample in Study 2a. Most notably, the differences between distraction and reappraisal were not significant in high or low-intensity conditions for the Indian sample, whereas in the U.S. these distinctions have been replicated in multiple studies. Thus, we decided to obtain a larger Indian sample and directly compare the results to a separate sample of American participants in Study 3. 


\section{STUDY 3}

After validating our experimental framework among both countries and detecting the presence of a preliminary culture effect, we sought to compare ER strategy choice between the two countries (Study 3a) and test whether it was in fact religious differences driving the effect (Study 3b).

\section{Study 3a}

In Study 3a, we compared ER choice between a new, larger Indian sample and a new American sample.

\section{Participants}

One hundred eight MTurk workers were recruited from either India ( $N=51, M_{\text {Age }}=34.14, S D_{\text {Age }}$ $=7.91)$ or the U.S. $\left(N=57, M_{\text {Age }}=36.70, S D_{\text {Age }}=11.78\right)$.

\section{Procedure}

The procedures were identical to those of Study 2.

\section{RESULTS}

Following the same procedures as Study 2, the number of erroneous double ER choice selections were calculated for all participants and participants were removed prior to conducting analyses if they had more than 2 of these mistakes. Four participants were removed from the Indian sample and zero participants were removed from the U.S. sample based on this standard. The numbers of failed manipulation checks were then calculated for each of the samples. Eighteen Indian participants were removed for having more than 2 failed manipulation checks and 5 U.S. participants were removed based on this criterion. These removals left a final sample of 29 Indiabased participants $\left(M_{\text {Age }}=34.93, S D_{\text {Age }}=8.24\right)$ and 52 US-based participants $\left(M_{\text {Age }}=37.38\right.$, $\left.S D_{\text {Age }}=12.09\right)$.

Table 3.1, ER Choice Cultural Comparison: Study 3a

\begin{tabular}{|l|l|l|l|l|}
\hline $\begin{array}{l}\text { High-Intensity } \\
\text { Images }\end{array}$ & \multicolumn{4}{|l|}{ U.S.A. } \\
\hline & $M$ & $95 \% C I$ & $M$ & $95 \% C I$ \\
\hline ER Choice & $22.07 \%$ & $14.28 \%-29.86 \%$ & $18.08 \%$ & $13.60 \%-22.55 \%$ \\
\hline Acceptance & $37.93 \%$ & $29.57 \%-46.29 \%$ & $55.38 \%$ & $50.21 \%-60.56 \%$ \\
\hline Distraction & $31.26 \%$ & $23.56 \%-38.97 \%$ & $20.90 \%$ & $17.26 \%-24.54 \%$ \\
\hline Reappraisal & $8.74 \%$ & $3.44 \%-14.03 \%$ & $5.64 \%$ & $1.57 \%-9.71 \%$ \\
\hline Watch & & & & \\
& & & & \\
& & &
\end{tabular}

(c) The International Journal of Indian Psychology, ISSN 2348-5396 (e)| ISSN: 2349-3429 (p) | 211 
Emotion Regulation Choice: Differences in U.S. and Indian Populations

\begin{tabular}{|c|c|c|c|c|}
\hline $\begin{array}{l}\text { Low-Intensity } \\
\text { Images }\end{array}$ & & & & \\
\hline & India & & U.S.A. & \\
\hline ER Choice & $M$ & $95 \% C I$ & $M$ & $95 \% C I$ \\
\hline Acceptance & $31.95 \%$ & $25.58 \%-38.33 \%$ & $30.38 \%$ & $24.52 \%-36.25 \%$ \\
\hline Distraction & $14.02 \%$ & $8.71 \%-19.33 \%$ & $8.97 \%$ & $6.38 \%-11.57 \%$ \\
\hline Reappraisal & $13.10 \%$ & $8.24 \%-17.97 \%$ & $22.05 \%$ & $16.59 \%-27.51 \%$ \\
\hline Watch & $40.92 \%$ & $31.15 \%-50.69 \%$ & $38.59 \%$ & $30.30 \%-46.88 \%$ \\
\hline
\end{tabular}

As illustrated in Table 3.1, there were stark differences between the ER choices of Indian and U.S. participants and these differences varied across image intensity. Most notably, the sharp distract in high-intensity/reappraise in low-intensity distinction that has been replicated in multiple Western-focused studies was not present in the Indian sample (Figure 3.1). Instead, there was no significant difference between Indian preference for distraction or reappraisal in either high, $t(28)=0.99, p=.332, d=.18$, or low-intensity conditions, $t(28)=0.24, p=.811, d$ $=.04$.

To investigate the differences between the U.S. and Indian samples, a three-way mixed factorial ANOVA was conducted with Image Intensity and ER Strategy as the within-subject factors and Country (India, US) as the between-subject factor. There was a significant Image intensity X ER Strategy X Country interaction, $F(3,237)=7.06, p<.001, \eta_{p}^{2}=.08$. Next, two-way mixed ANOVAs were conducted to examine the Country X ER Strategy Interaction for the highintensity and low-intensity images separately. For the high-intensity images, there was a significant Country X ER Strategy interaction, $F(3,237)=6.95, p<.001, \eta_{p}^{2}=.08$, whereas the Country X ER Strategy interaction was not significant when only examining the low-intensity images, $F(3,237)=1.30, p>.05, \eta_{p}^{2}=.02$. Independent sample t-tests were conducted to compare the ER strategy usage between countries in high-intensity conditions. Indian participants reported using distraction less frequently than U.S. participants while viewing highintensity images, $t(79)=-3.79, p<.001, d=.88$. Indian participants reported using reappraisal more frequently than U.S. participants when presented with high-intensity images, $t(79)=2.80, p$ $=.006, d=.65$. There were no country differences in watch for high-intensity images and contrary to our hypothesis, acceptance usage for high-intensity images also did not differ significantly by country, both $t$ 's $<.97$, both $p$ 's $>.3$, both $d$ 's $<.23$. The omnibus Country X ER Strategy interaction was not significant for low-intensity images. However, an independent sample t-test indicated that for low-intensity images, U.S. participants did reappraise significantly more than Indian participants, $t(79)=-2.21, p=.030, d=.51$. 
Figure 3.1 Reappraisal Minus Distraction Within-Group Differences

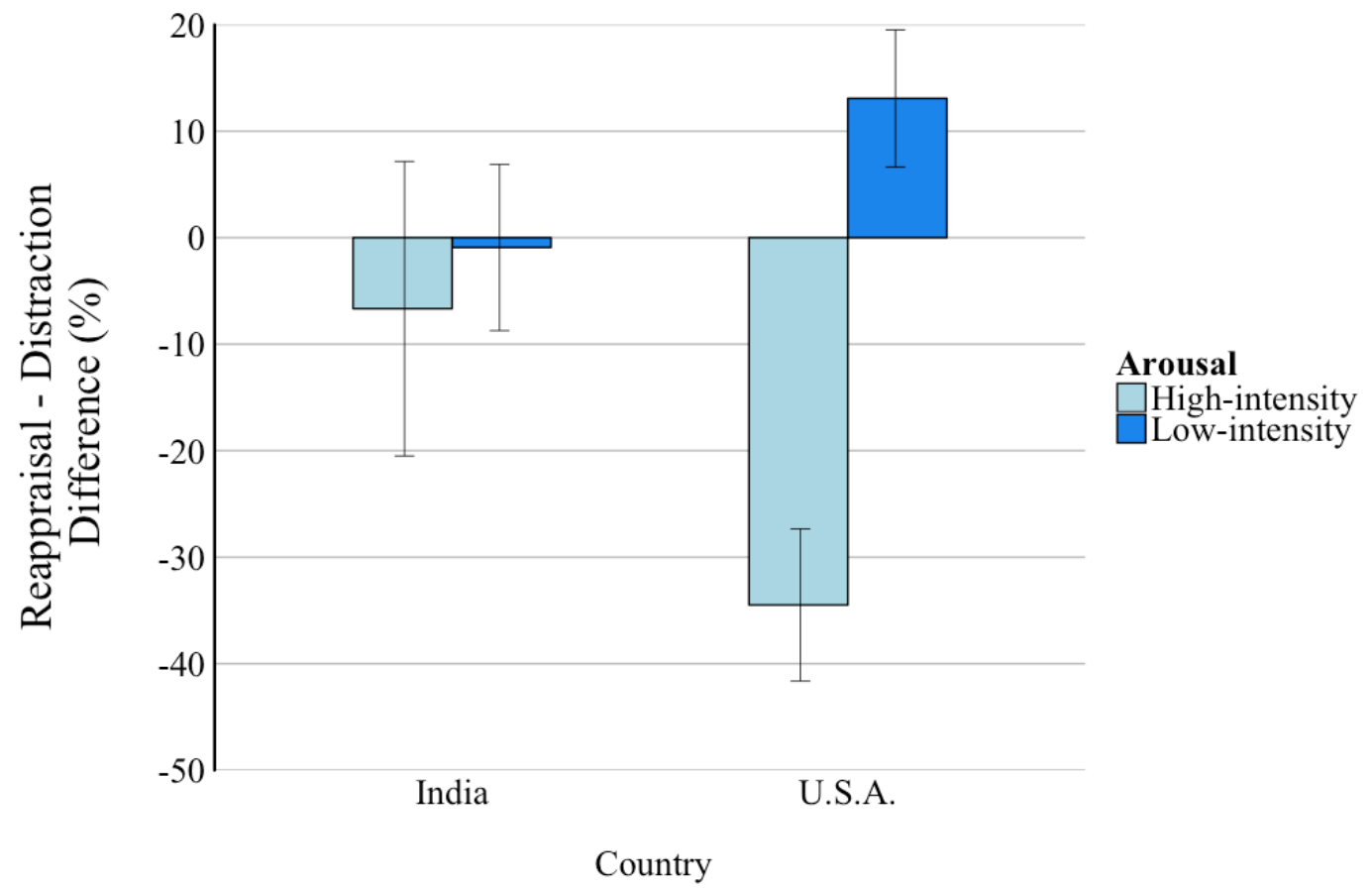

\section{DISCUSSION}

The results of Study 3a supported our prediction that ER choices are moderated by culture, as demonstrated by the significant Intensity X ER Strategy X Country 3-way interaction. Contrary to our expectations, we found that being raised in a culture that espouses a spiritually founded philosophy of acceptance and displays higher rates of fatalistic outlooks does not appear to increase acceptance usage as an emotion regulation strategy. Our prediction that Indian participants would use reappraisal more due to their greater religiosity was supported in the highintensity condition. However, the U.S. sample reappraised more frequently than the Indian sample for low-intensity images. We believed that religiosity was one cultural feature driving the elevated rates of reappraisal in high-intensity conditions, but wanted to investigate this hypothesis further in Study 3b.

\section{Study $3 b$}

In Study 3b, we sought to determine if Indian participants were in fact interpreting and reappraising images through a religious framework. To do this, we ran another online crosscultural comparison survey in which we asked new participants to write either reappraisals or descriptions of the same 30 images used in prior studies. We hypothesized that Indian participants would more frequently incorporate religious themes in their responses to the images and more frequently use those themes to formulate reappraisals. 


\section{Emotion Regulation Choice: Differences in U.S. and Indian Populations}

\section{Participants}

One hundred three MTurk workers were recruited from either India ( $N=52, M_{\text {Age }}=34.35, S D_{\text {Age }}$ $=10.01)$ or the U.S. $\left(N=51, M_{\text {Age }}=35.99, S D_{\text {Age }}=9.72\right)$.

\section{Procedure}

First, participants were given a brief introduction to what reappraisal was. Next, they were presented with a negatively valenced image and given an example on what a reappraisal of that image might be. After two of such training exercises, the experiment began. Participants were presented with the same 30 images used in prior studies and asked to do three things. (1) They were asked to rate the intensity of negative emotion they felt in response to the image on a scale of 1 (little to no negative emotion) to 9 (extreme negative emotion). (2) They were asked if they were able to think of a reappraisal for the image (yes/no). (3) If yes, they were asked to write a 23 sentence reappraisal of the image. If no, they were asked to write a 2-3 sentence description of the image. Image order in the training segment and the experiment segment was randomized.

\section{RESULTS}

To compare the high and low-intensity image ratings between Indian and U.S. participants, independent sample t-tests were conducted with Bonferroni corrections. Indian participants reported feeling higher intensity emotion in response to low-intensity images $\left(M_{\text {low }}=4.50 ; S D_{\text {low }}\right.$ $=0.84)$ than U.S. participants $\left(M_{\text {low }}=3.73 ; S D_{\text {low }}=0.55\right), t(28)=-2.99, p=.011, d=1.09$. Indian participants emotional intensity in response to high-intensity images $\left(M_{\text {high }}=7.02 ; S D_{\text {high }}\right.$ $=1.04$ ) was not significantly different than U.S. participants $\left(M_{\text {high }}=6.76 ; S D_{\text {high }}=0.91\right), t(28)=$ $-0.73, p=.940, d=.27$. Our hypothesis that the Indian sample would incorporate religion more frequently in this exercise was supported. We operationalized this criterion as a participant's response including at least 1 of the 3 words; "god", "soul”, or "bless". Upon examining the data, we found that 13 Indian participants incorporated religious themes in their responses or used religion directly as a framework to formulate reappraisals, while in the U.S. sample only 2 participants fit this criterion, $\chi^{2}(1)=7.58, p=.006$. For example, in response to a picture of a group of crying people at what appears to be a funeral, one Indian participant commented that the deceased was “going to God”. In response to a picture of piled skulls, an Indian participant responded that their souls were in heaven. One American participant commented that a man in prison had found God.

\section{DISCUSSION}

The difference in religious themes present in people's responses to the photos further supports our hypothesis that religion is a facet of Indian culture that contributes to their more frequent reappraisal choice in a high-intensity context. Some of these responses were reappraisals that were afforded by beliefs about the afterlife. Others expected God to remedy the problems faced in the photograph. Others still were simply supplications for God to intervene. American nonreligious reappraisals often involved potential mitigating factors that were not immediately 


\section{Emotion Regulation Choice: Differences in U.S. and Indian Populations}

apparent. Other American reappraisals involved hope for the future-oftentimes regarding help from other people that the subject of the image would receive. Indian participants also reappraised in some instances by discussing the help that the subject of the image would receive from other people. However, when the subject appeared beyond the aid of humans, God was often there to pick up the slack.

\section{General Discussion}

In our present research, we first designed and piloted an experimental structure to test participants' ER choices between three common strategies; reappraisal, acceptance, and distraction (Studies 1 and 2). Next, we used this test to compare ER choice frequencies between two highly populous, yet notably dissimilar countries in high and low-intensity negative conditions (Study 3). As anticipated by our first hypothesis, Indian people were significantly more prone to use reappraisal in response to high-intensity negative stimuli than people from the U.S.A. This may be due to Indian religiosity offering satisfactory reappraisal affordances in high-intensity situations where otherwise, there were few. Contrary to our second hypothesis, Indian participants did not show greater preference for an acceptance ER strategy than American participants.

This research demonstrates that the dominant modes of thought that are shaped by culture influence ER choices differently across varying contexts. These culturally driven differences can be traced at least in part, to the differing content of cognitive reappraisals. Since reappraisal involves actively changing the meaning of events, culturally specific meaning systems such as religion are a fundamental ingredient of this process.

The discovery of cultural differences in ER choice aligns well with the shift from the traditional approach of labeling strategies adaptive or maladaptive, into more recent research that places an emphasis on context-specific ER flexibility (Bonanno, Papa, Lalande, Westphal, \& Coifman, 2004; Sheppes et al., 2014). Cultural differences in ER choice suggest that universally "normal" or adaptive strategy selection does not exist. However, the ability to flexibly choose between strategies in response to different situations does appear to be consistent across cultural lines.

This finding has important clinical implications. The DSM-5 (2013) explicitly states that culturally approved responses to stressors are not classified as mental disorders. Thus, medical practitioners must be aware of cultural variation in ER choice in order to accurately diagnose and administer care to non-Western patients. Additionally, if certain cultural attributes facilitate certain ER strategies, clinicians can encourage a focus on these cultural attributes for patients when it is adaptive. For example, if religiosity facilitates reappraisal, clinicians can encourage attention towards the patient's religious beliefs in situations that require repeated engagement and thus, could benefit from reappraisal (Kross, \& Ayduk, 2008). 


\section{Emotion Regulation Choice: Differences in U.S. and Indian Populations}

One limitation of this series of studies is that by surveying English-speaking Indian participants over the internet, our samples were limited to Indian people with ample exposure to Western culture. We believe that if we had access to Indian participants in more rural segments of the population, we may have obtained an even stronger effect. A second limitation of our study is that although we demonstrated a general cultural effect and pointed to one cultural attribute, there are likely multiple drivers that contribute to cross-cultural differences in ER choice. Future cross-cultural studies of ER choice are needed to isolate other specific cultural determinants. Another limitation of the present study is that differences in initial appraisal were not addressed. Prior studies have demonstrated that there is some cultural variability in how people appraise situations (Mesquita \& Frijda, 1992; Scherer, 1997; Imada \& Ellsworth, 2011). These culturally variant appraisals could factor into different ER choices. Further studies would be required to investigate the role that varying appraisals play into culturally variant ER choice.

While one study series cannot summarize the entirety of ER choice differences between two of the largest and most diverse cultures on Earth, this research suggests that ER choices differ across culture and are at least in part, tied to religiosity. That said, India is an extraordinarily populous country and sweeping generalizations cannot be made based on a limited sample size. The next step for future studies would be to narrow down subcultural groups within these broader categories and pinpoint additional ingrained modes of thought that are driving ER strategy selection.

\section{Notes}

1. For copyright reasons, images used in Figure 2.1 are similar, but not identical to low-intensity images used in the experiment.

\section{Acknowledgments}

The author appreciates all those who participated in the study and helped to facilitate the research process.

\section{Conflict of Interests:}

The author declared no conflict of interests. 


\section{REFERENCES}

American Psychiatric Association. (2013). Diagnostic and statistical manual of mental disorders (5th ed.). Arlington, VA: American Psychiatric Publishing.

Aycan, A. (2000). Impact of Culture on Human Resource Management Practices: A 10-Country Comparison. Applied Psychology: An International Review, 49, 192-221.

Bechara, A., Damasio, H., Tranel, D., Damasio, A.R. (1997). Deciding Advantageously Before Knowing the Advantageous Strategy. Science, 275, 1293-1295.

Bernstein, M.H. (1992). Fatalism. Lincoln: University of Nebraska Press.

Bonanno, G. A., Papa, A., Lalande, K., Westphal, M., \& Coifman, K. (2004). The importance of being flexible: The ability to both enhance and suppress emotional expression predicts long-term adjustment. Psychological Science, 15, 482-487.

Bradley M. M., Codispoti, M., Cuthbert, B. N., \& Lang, P. J. (2001). Emotion and motivation I: Defensive and appetitive reactions in picture processing. Emotion, 1, 276-298.

Butler, E., Lee, T., \& Gross, J.J. (2007). Emotion regulation and culture: Are the social consequences of emotion suppression culture-specific?. Emotion, 7, 30-48.

C-1 Population by Religious Community. (2011). Office of the Registrar General \& Census Commissioner. Retrieved from http://www.censusindia.gov.in/2011census/C-01.html

Crabtree, S. (2010). Religiosity Highest in World's Poorest Nations. Retrieved from http://www.gallup.com/poll/142727/religiosity-highest-world-poorest-nations.aspx

Elderly Patients: A Two-year Longitudinal Study. Journal of Health Psychology, 9, 713-730.

English, T., John, O. P., Srivastava, S., \& Gross, J.J. (2012). Emotion regulation and peer-rated social functioning: A 4-year longitudinal study. Journal of Research in Personality, 46, 784.

Gainesville: University of Florida. Matsumoto, D., Yoo, S.H., \& Nakagawa, S. (2008). Journal of Personality and Social Psychology, 94, 925-937.

Gross, J. J. (2002). Emotion regulation: Affective, cognitive, and social consequences. Psychophysiology, 39, 281-291.

Gross, J.J., \& John, O. P. (2003). Individual differences in two emotion regulation processes: Implications for affect, relationships, and well-being. Journal of Personality and Social Psychology, 85, 348-362.

Gross, J.J., \& Muñoz, R.F. (1995). Emotion regulation and mental health. Clinical Psychology: Science and Practice, 2, 151-164.

Hay, A. C., Sheppes, G., Gross, J., \& Gruber, J. (2015). Choosing how to feel: Emotion regulation choice in bipolar disorder. Emotion, 15, 139-145.

Hayes, S. C., Strosahl, K., \& Wilson, K. G. (1999). Acceptance and Commitment Therapy: An experiential approach to behavior change. New York, NY: Guilford Press.

Imada, T., \& Ellsworth, P. (2011). Proud Americans and lucky Japanese: Cultural differences in appraisal and corresponding emotion. Emotion, 11, 329-345. 


\section{Emotion Regulation Choice: Differences in U.S. and Indian Populations}

John, O. P., \& Gross, J. J. (2004). Healthy and unhealthy emotion regulation: Personality processes, individual differences, and lifespan development. Journal of Personality, 72, 1301-1334.

Keltner, D., Haidt, J. (1999). Social Functions of Emotions at Four Levels of Analysis. Cognition and Emotion, 13, 505-521.

Koopmann-Holm, B. \& Tsai, J.L. Focusing on the Negative: Cultural Differences in Expressions of Sympathy. Journal of Personality and Social Psychology, 107, 1092-1115.

Kross, E., \& Ayduk, O. (2008). Facilitating adaptive emotional analysis: Distinguishing distanced-analysis of depressive experiences from immersed-analysis and distraction. Personality and Social Psychology Bulletin, 34, 924-938.

Kwon, H., Yoon, K. L., Joormann, J., \& Kwon, J. (2013). Cultural and gender differences in emotion regulation: Relation to depression. Cognition and Emotion, 27, 769-782.

Lang, P. J., Bradley, B. N., \& Cuthbert, B. N. (2008). International Affective Picture System (IAPS): Affective ratings of pictures and instruction manual (Technical Report No. A-8).

Mesquita, B. \& Frijda, N.H. (1992). Cultural Variations in Emotion: A Review. Psychological Bulletin, 112, 179-204.

Pargament, K. I., Koenig, H. G., Tarakeshwar, N., \& Hahn, J. (2004). Religious Coping Methods as Predictors of Psychological, Physical and Spiritual Outcomes among Medically Ill

Park, C.L. (2005) Religion as a Meaning-Making Framework in Coping with Life Stress. Journal of Social Issues, 61, 707-729.

Richards, J., \& Gross, J. J. (2000). Emotion regulation and memory: The cognitive costs of keeping one's cool. Journal of Personality and Social Psychology, 79, 410-424.

Safran, W., Sahoo, A. K., \& Lal, B. V. (2008). Indian Diaspora in Transnational Contexts: Introduction. Journal of Intercultural Studies, 29, 1-5.

Scheibe, S., Sheppes, G. \& Staudinger, U. (2015). Distract or reappraise? Age-related differences in emotion-regulation choice. Emotion, 15, 677-681.

Scherer, K. (1997). The role of culture in emotion-antecedent appraisal. Journal of Personality and Social Psychology, 73, 902-922.

Shafir, R., Schwartz, N., Blechert, J., \& Sheppes, G. (2015). Emotional intensity influences preimplementation and implementation of distraction and reappraisal. Social Cognitive and Affective Neuroscience, 10, 1329-1337.

Sheppes, G., Scheibe, S., Suri, G., \& Gross, J. J. (2011). Emotion regulation choice. Psychological Science, 22, 1391-1396.

Sheppes, G., Scheibe, S., Suri, G., Radu, P., Blechert, J. \& Gross, J.J. (2014). Emotion Regulation Choice: A Conceptual Framework and Supporting Evidence. Journal of Experimental Psychology: General, 143, 163-181.

Soto, J.A., Perez, C.R., Kim, Y., Lee, E.A. Minnick, M.R. (2011) Is expressive suppression always associated with poorer psychological functioning? A cross-cultural comparison between European Americans and Hong Kong Chinese. Emotion, 11, 1450-1455. 
Suri, G., Sheppes, G., Young, G., Abraham D., McRae, K., \& Gross, J.J. (in press). Emotion Regulation Choice: The Role of Environmental Affordances.

Susskind, J., Lee, D., Cusee, A., Feiman, R., Grabski, W., \& Anderson, A. (2008) Expressing fear enhances sensory acquisition. Nature Neuroscience, 11, 843-850.

Vishkin, A., Bigman, Y. E., Porat, R., Solak, N., Halperin, E., \& Tamir, M. (2016). God rest our hearts: Religiosity and cognitive reappraisal. Emotion, 16, 252-262.

Weber, E.U., Hsee, C.K. (2000). Culture and Individual Judgement and Decision Making. Applied Psychology: An International Review, 49, 32-61.

Weinberg, A., \& Hajcak, G. (2010). Beyond good and evil: The time course of neural activity elicited by specific picture content. Emotion, 10, 92-100.

Whitman, S. M. (2007). Pain and Suffering as Viewed by the Hindu Religion. The Journal of Pain, 8, 607-613.

World Population Data Sheet. (2016). Population Reference Bureau. Retrieved from http://www.prb.org/pdf16/prb-wpds2016-web-2016.pdf

Yates, J.F., \& Oliveira, S. (2016). Culture and Decision Making. Organizational Behavior and Human Decision Processes, 136, 106-118.

How to cite this article: Mehta A, Young G, Wicker A, Barber S, Suri G (2017), Emotion Regulation Choice: Differences in U.S. and Indian Populations, International Journal of Indian Psychology, Volume 4, Issue 2, No. 94, ISSN:2348-5396 (e), ISSN:2349-3429 (p), DIP:18.01.160/20170402, ISBN:978-1-365-84229-0 